\title{
Diffusion tensor imaging of renal parenchyma in pediatric patients with chronic kidney disease: Correlation with serum biomarkers
}

\author{
Ahmed Abdel Razek ${ }^{1}$, Ayman Hammad², Manar Mansour², Marwa Ramadan², Khadiga \\ $\mathrm{Ali}^{2}$, Dina Ibrahim ${ }^{2}$, and Tarek Barakat ${ }^{2}$ \\ ${ }^{1}$ Mansoura University Faculty of Medicine \\ ${ }^{2}$ Affiliation not available
}

February 3, 2021

\begin{abstract}
Purpose: to demonstrate role of diffusion tensor imaging (DTI) in diagnosis of pediatric chronic kidney disease (CKD) using fraction anisotropy (FA) and apparent diffusion coefficient (ADC). Material and methods: Prospective study done on 35 CKD patients (19 boys, 16 girls; mean age $12.2 \pm 2.7$ years) and 19 age and sex-matched volunteers. Patients with sclerotic $(\mathrm{n}=25)$ and non-sclerotic $(\mathrm{n}=10)$ CKD that underwent DTI of kidney. Results: Mean FA of renal cortex/ medulla in CKD $(0.20 \pm 0.07$, and $0.18 \pm 0.08)$ was significantly lower $(\mathrm{p}=0.001)$ from volunteers $(0.27 \pm 0.08,0.31 \pm 0.09)$. Cutoff renal FA of cortex/ medulla used for diagnosis of CKD was 0.23 , and 0.22 with AUC of $0.828,0.828$ and accuracy of $82.9 \%, 80.7 \%$. Mean ADC of renal cortex/ medulla in CKD $(1.98 \pm 0.23$ and $2.03 \pm 0.23 \mathrm{X} 10-3 \mathrm{~mm} 2 / \mathrm{s})$ was significantly higher $(\mathrm{p}=0.001)$ that of volunteers $(1.65 \pm 0.134$ and $1.68 \pm 0.16 \mathrm{X} 10-3 \mathrm{~mm} 2 / \mathrm{s}$. Cutoff renal ADC of cortex/medulla used to diagnosis of CKD were $1.75 \mathrm{and} 1.85 \mathrm{X} 10-3 \mathrm{~mm} 2 / \mathrm{s}$ with AUC of $0.828,0.910,0.828$ and 0.81 and accuracy of $82.9 \%, 84.1 \%, 80.7 \%$ and $79.5 \%$. FA of renal cortex/medulla in sclerotic CKD was significantly different $(\mathrm{p}=0.001)$ than non-sclerotic CKD $(0.26 \pm 0.07$ and $0.25 \pm 0.08)$. The FA of renal cortex/medulla in CKD patients correlated with serum creatinine $(\mathrm{r}=-0.468 ; \mathrm{p}=0.000, \mathrm{r}=-0.381 ; \mathrm{p}=0.001)$, e GFR $(\mathrm{r}=$ $0.364 ; \mathrm{p}=0.002, \mathrm{r}=0.318 ; \mathrm{p}=0.007)$. Conclusion: FA and ADC of renal cortex/ medulla can help in diagnosis of CKD, FA cortex/ medulla predicts sclerotic CKD and correlated with some of serum biomarkers.
\end{abstract}

\section{Hosted file}

2-Renal paper9Clin.pdf available at https://authorea.com/users/393481/articles/507092diffusion-tensor-imaging-of-renal-parenchyma-in-pediatric-patients-with-chronic-kidneydisease-correlation-with-serum-biomarkers 

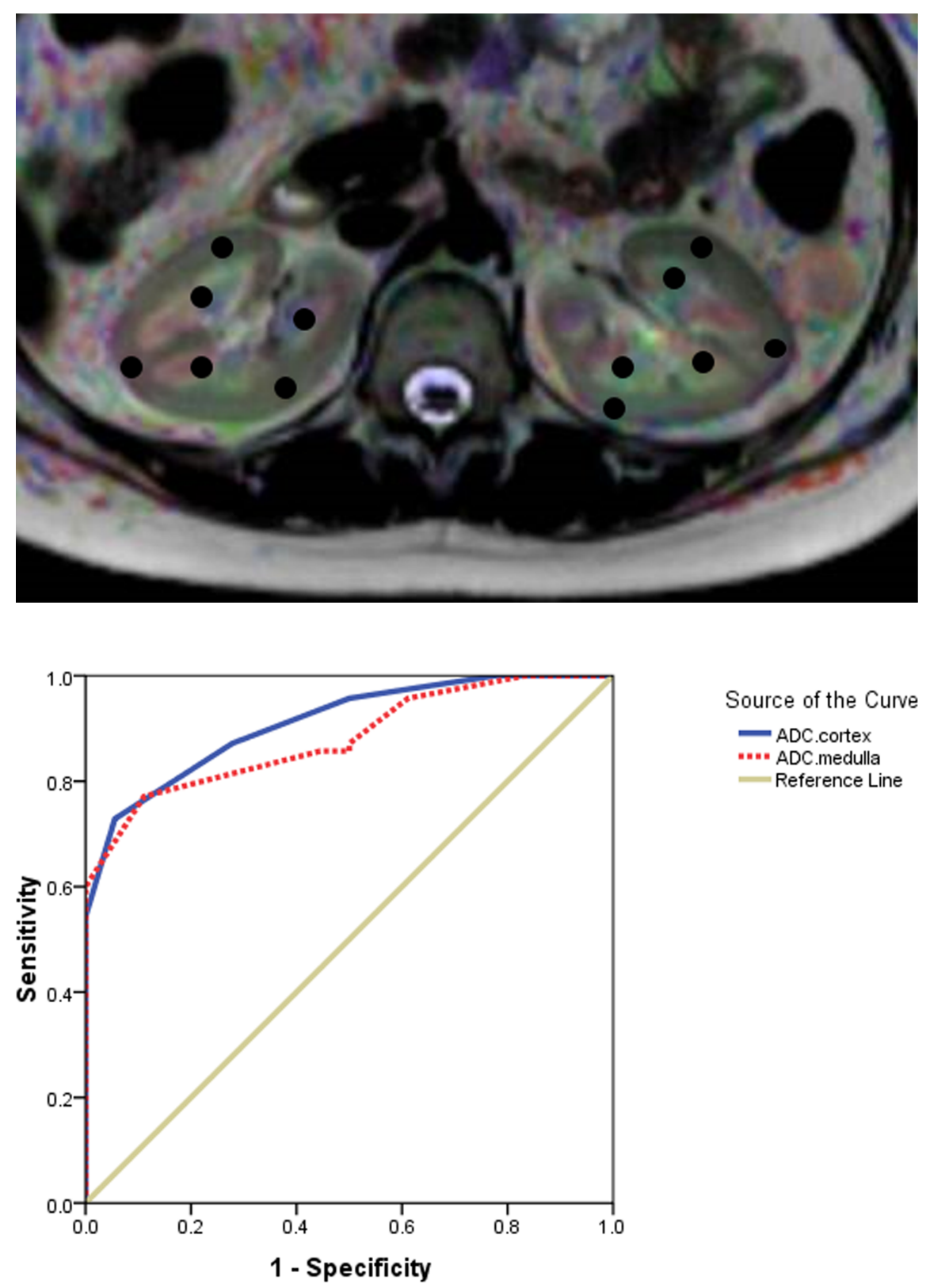

Source of the Curve

ADC.cortex

" " ADC medulla

- Reference Line 


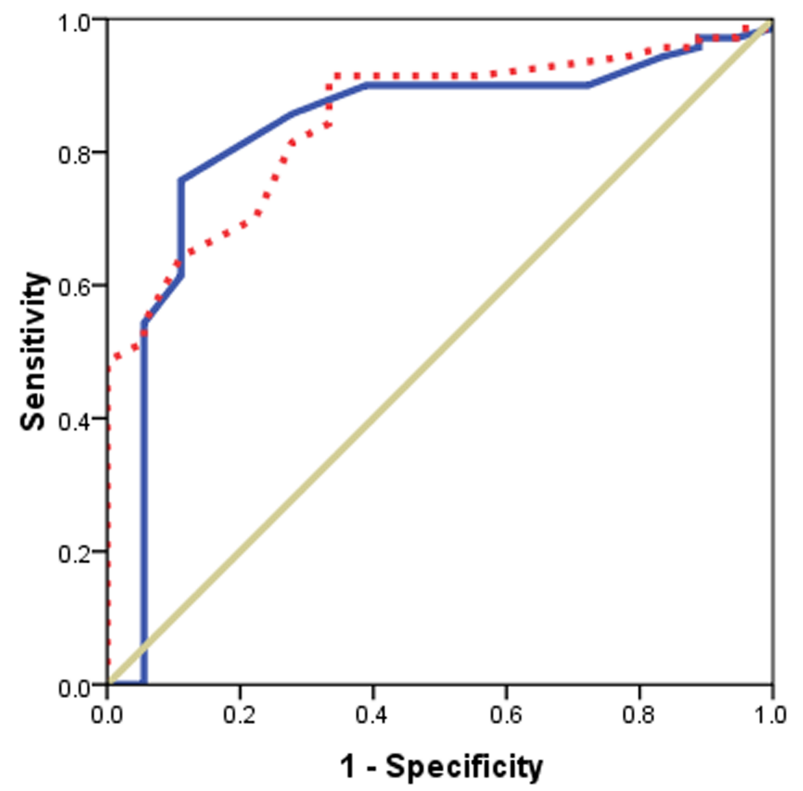

Source of the Curve

FA.cortex

- 'Fa.medualla

- Reference Line

1 - Specificity 